\title{
Brainstem angiocentric glioma: report of 2 cases
}

\author{
Kristin J. Weaver, MD, PhD, ${ }^{1}$ Lexi M. Crawford, BS, ${ }^{1}$ Jeffrey A. Bennett, MD, ${ }^{2}$ \\ Marie L. Rivera-Zengotita, MD, ${ }^{3}$ and David W. Pincus, MD, PhD' \\ Departments of ${ }^{1}$ Neurosurgery, ${ }^{2}$ Radiology, and ${ }^{3}$ Pathology, University of Florida, Gainesville, Florida
}

\begin{abstract}
Angiocentric glioma is a rare tumor that was recognized by the WHO Classification of Tumours of the Central Nervous System as a distinct clinicopathological entity in 2007. Since this initial description, the vast majority of cases of angiocentric glioma reported in the literature have involved tumors of the cerebral hemispheres. To date, only 1 case of angiocentric glioma arising from the posterior midbrain has been reported. The authors present the cases of 2 pediatric patients who were found to have brainstem angiocentric gliomas. The clinical course, radiological and pathological features, treatment, and follow-up are described. The first case is one of a 5-year-old girl who presented with double vision, headache, and nausea and was found to have a midbrain lesion with pathological features consistent with angiocentric glioma. She was treated with resection and endoscopic third ventriculostomy (ETV), followed by close observation and serial neuroimaging. The second case is one of a 6 -year-old boy who presented with progressive mouth drooping and problems with balance. He was found to have a pontine lesion with pathological features consistent with angiocentric glioma. This patient was treated with ETV, followed by close observation and serial neuroimaging. This report includes 6 and 1.5 years of follow-up of the patients, respectively. While there are limited data regarding the prognosis or long-term management of patients with brainstem angiocentric gliomas, the cases described in this report suggest an indolent course for this tumor, similar to the course of angiocentric gliomas located in the cerebral hemispheres.
\end{abstract}

https://thejns.org/doi/abs/10.3171/2017.5.PEDS16402

KEY WORDS angiocentric glioma; brainstem; oncology; pediatric neurosurgery

A NGIOCENTRIC glioma, first identified in 2005 , is a rare type of brain tumor that predominantly affects children and young adults. ${ }^{49}$ In 2007, the WHO Classification of Tumours of the Central Nervous System ${ }^{5}$ officially recognized this tumor as a distinct clinicopathological entity where it was grouped with astroblastoma and chordoid glioma of the third ventricle in the category of "other neuroepithelial tumours." It was classified as WHO Grade I due to its benign clinical behavior and possibility for a surgical cure. ${ }^{1}$ To date, approximately 65 cases of angiocentric glioma have been reported in the literature. ${ }^{6} \mathrm{~A}$ majority of the cases exhibit similar MRI features as well as histopathological characteristics. MRI features include hyperintensity on T2-weighted and FLAIR images without contrast enhancement. ${ }^{6}$ Histopathological characteristics include monomorphous bipolar cells, an angiocentric growth pattern, and features of ependymal differentiation with dot-like staining for epithelial membrane antigen (EMA). In addition, a majority of the tumor cells exhibit immunoreactivity for GFAP, S100 protein, and vimentin but not for neuronal antigens. ${ }^{1,6}$
The vast majority of angiocentric gliomas are located supratentorially, with the most common tumor locations including the frontoparietal cortex and the temporal lobe, specifically the hippocampal region. A majority of patients present with refractory epilepsy as the chief complaint. ${ }^{6}$ To date, only 1 case of angiocentric glioma located in the brainstem has been reported in a 3 -year-old patient. ${ }^{3}$ In this report, we present the unique cases of 2 pediatric patients diagnosed with angiocentric glioma localized to the brainstem. The clinical course, radiological and pathological features, treatment, and follow-up are described, with the goal of adding to the limited knowledge that exists on the characteristics, treatment, and prognosis of brainstem angiocentric glioma.

\section{Case Reports \\ Case 1}

History, Examination, and Operation

A previously healthy 5-year-old girl presented with a 1-week history of double vision, headache, and nausea. 

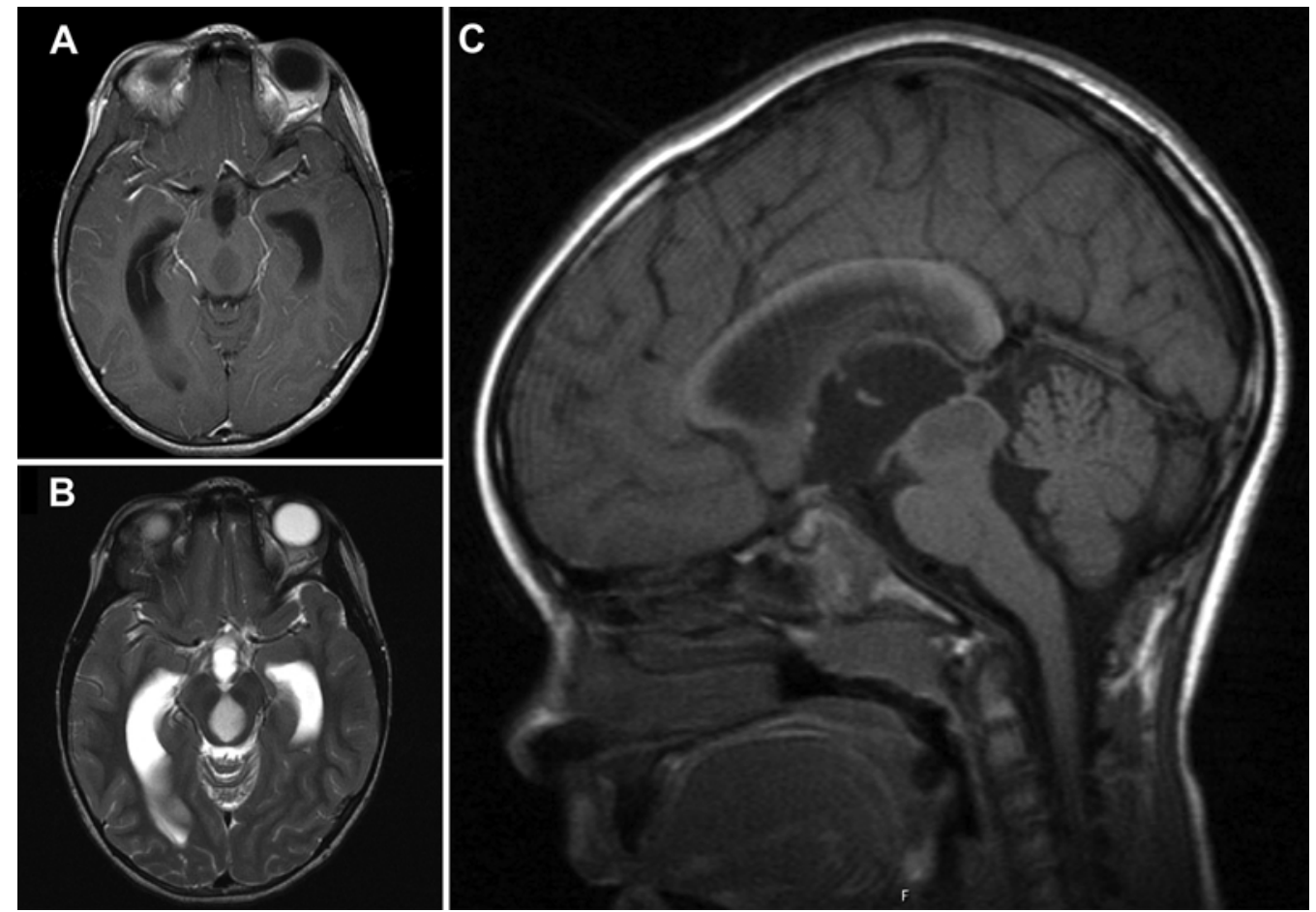

FIG. 1. Case 1. A: Axial T1-weighted MR image with contrast demonstrating a nonenhancing lesion of the midbrain with low T1 signal. The lesion measures $1.2 \times 1.8 \times 1.1 \mathrm{~cm}$. There is obstruction of the aqueduct with associated ventriculomegaly. B: Axial T2-weighted MR image demonstrating high T2 signal of the midbrain lesion. C: Sagittal T1-weighted MR image.

Physical examination revealed a left abducens nerve palsy and papilledema. Brain MRI revealed a nonenhancing midbrain lesion with low T1 signal (Fig. 1A and C) and high T2 signal (Fig. 1B) that was causing aqueductal obstruction and ventriculomegaly. A small portion of the aqueduct could be seen posterior to the lesion; therefore, the lesion was considered a tegmental lesion rather than a tectal lesion. After undergoing stereotactic endoscopic biopsy of the midbrain lesion and endoscopic third ventriculostomy (ETV), the patient was observed with serial imaging and ophthalmological examinations. She remained stable until 6 months after the initial diagnosis, when she developed progression of symptoms, specifically development of dorsal midbrain syndrome. MRI at that time revealed a $20 \%$ increase in tumor size. She was then treated for 6 months with a monthly dose of carboplatin, which unfortunately yielded little therapeutic effect. The decision was then made to perform a craniotomy for tumor resection. A posterior, interhemispheric transcallosal approach with microsurgical and stereotactic volumetric near-total resection of the midbrain tumor was completed.

\section{Pathological Examination}

Pathological examination of the initial biopsy specimen revealed a low-grade glial neoplasm with ependymal and astrocytic features and low Ki-67 proliferation index. The tumor was classified as a low-grade glioma (WHO Grade II). Histological study of the resected specimen revealed a glial neoplasm composed of a monomorphic population of tumor cells with "crisp" nuclear chromatin and mini- mal anaplasia, which has been recently described in the angiocentric glioma (Fig. 2A). The tumor demonstrated a prominent "angiocentric" growth pattern (Fig. 2B) and strong, especially perivascular, reactivity for GFAP (Fig. 2C). Dot-like EMA immunoreactivity was focally present (Fig. 2D). Neurofilament protein (RMdO 20) and synaptophysin demonstrated mature (presumably native) neurons and axonal processes but were negative in tumor cells. Mitotic activity was not readily identified, and the Ki-67 labeling index was approximately 5\%. No evidence of microvascular proliferation or necrosis was identified. The tumor was diagnosed as a low-grade glioma with features consistent with angiocentric glioma. Molecular testing was negative for $B R A F V 600 E$ mutation. Our pathologist reviewed the original biopsy specimen at the time of the resection specimen and concluded it was identical.

\section{Postoperative Course}

The initial postoperative course was complicated by altered mental status, cranial neuropathy, and gait abnormalities; however, the patient's neurological examination findings and functional status vastly improved in the months after surgery with the help of physical and occupational rehabilitation. After resection and analysis of the pathological specimen, no further chemotherapy or radiotherapy was recommended. The patient has been observed with yearly MRI studies for 6 years, with the most recent MRI study revealing minimal, stable residual brainstem tumor (Fig. 3). At her most recent visit, the patient's neurological examination showed stable cranial neuropathies and gait abnormalities. 


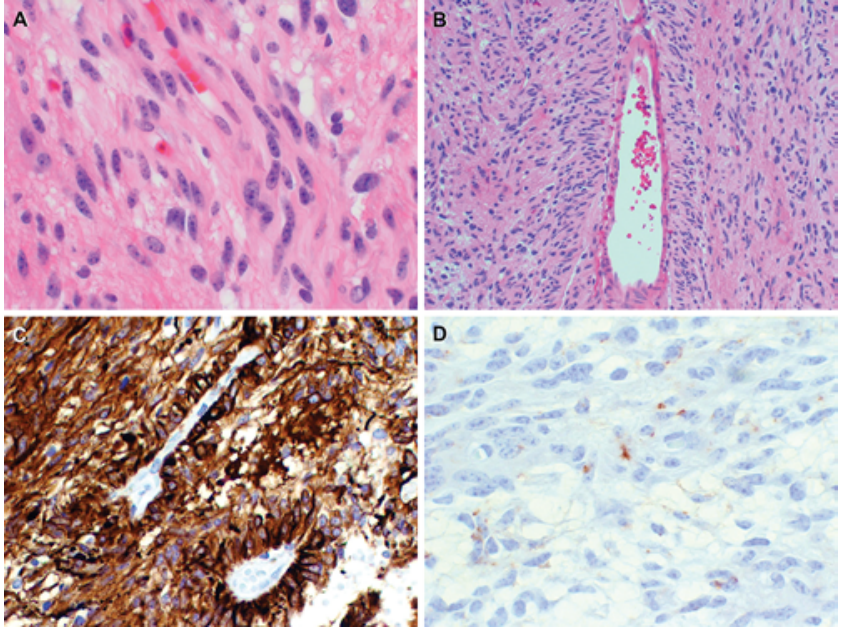

FIG. 2. Case 1. A: Photomicrograph showing individual tumor cells with "crisp" nuclear chromatin. B: Photomicrograph showing a prominent angiocentric growth pattern of the tumor cells. C: Photomicrograph showing tumor cells with strong positivity for GFAP, most prominently in the perivascular region. D: Photomicrograph showing tumor cells with perinuclear dot-like reactivity to EMA. H\& E (A and B). Original magnification $\times 400(A$ and $D), \times 100(B$ and $C)$. Figure is available in color online only.

\section{Case 2}

History, Examination, and Operation

A previously healthy 6-year-old boy presented with mouth drooping progressing over 1.5 years and issues with balance progressing over a period of 6 months. Physical examination revealed mild hemiparetic gait and left facial nerve palsy. Brain MRI revealed a diffuse nonenhancing pontine lesion with a large exophytic component projecting into the rostral fourth ventricle, producing only minimal ventricular enlargement. The lesion also extended into the right inferior and the right middle cerebellar peduncles. Imaging features were consistent with a diffuse pontine glioma (Fig. 4). The patient underwent a stereotactic biopsy of the lesion and ETV.

\section{Pathological Findings}

Microscopic examination of the biopsy specimen revealed a tumor with a distinctive angiocentric growth pattern composed of monomorphic round to spindled tumor cells displaying "crisp" nuclear chromatin (Fig. 5A). Tumor cells and tumor cell processes were diffusely reactive for GFAP (Fig. 5B). Dot-like EMA (Fig. 5C) and CD99 (Fig. 5D) reactivity were present in the tumor cells. Neurofilament protein (RMdO 20) showed evidence of mature infiltrated neurons and axonal processes but was negative in tumor cells. Mitosis, microvascular proliferation, and necrosis were not identified. The Ki-67 labeling index was low (approximately 3\%). The tumor was IDHI negative. A diagnosis of low-grade glial neoplasm with features of angiocentric glioma was rendered. The tumor was negative for BRAF V600E mutation.

\section{Postoperative Course}

The patient has undergone follow-up with serial imag-
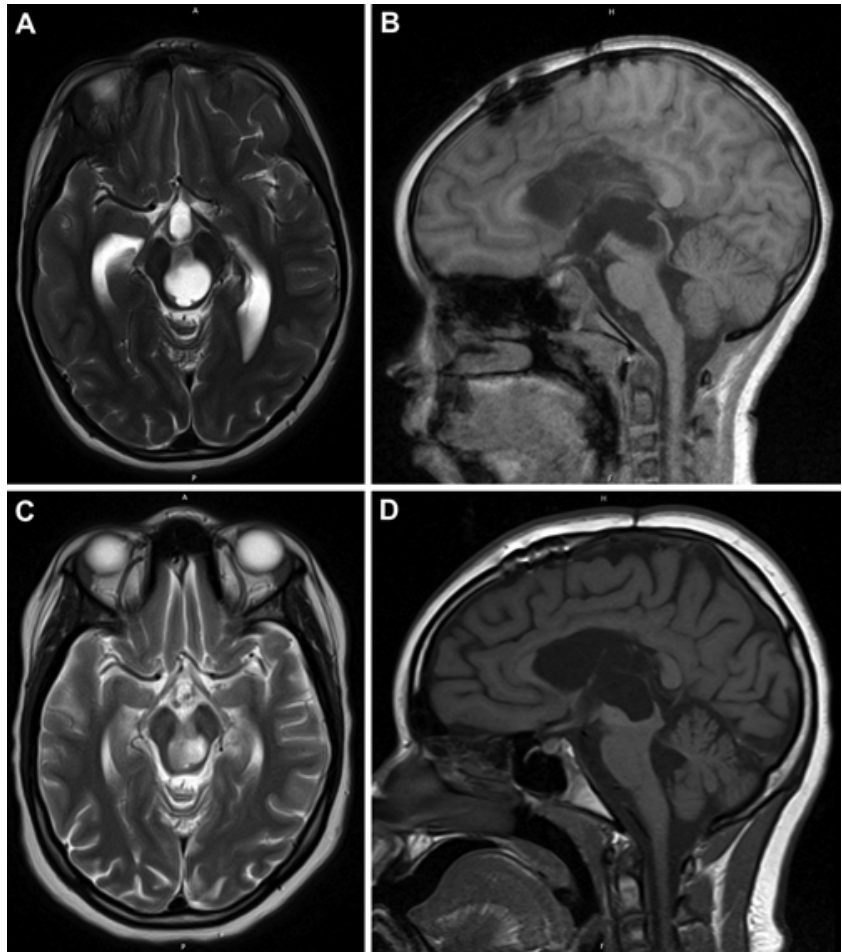

FIG. 3. Case 2. Axial T2-weighted (A) and sagittal T1-weighted (B) MR images obtained after biopsy, showing interval growth of tumor. Axial T2-weighted (C) and sagittal T1-weighted (D) MR images obtained after subtotal resection.

ing for 1.5 years with no radiographic evidence of progression.

\section{Discussion}

Angiocentric glioma is a rare, low-grade brain tumor that was first described in 2005 in 2 separate series that included 18 cases in total. ${ }^{4,9}$ Following these original descriptions, the tumor was officially recognized by the WHO Classification of Tumours of the Central Nervous System in 2007 and placed in the category of "other neuroepithelial tumours." It was classified as WHO Grade I due its indolent clinical behavior, absent mitoses, low proliferation index, and possibility for a surgical cure via resection. ${ }^{1}$ A review of the literature revealed 65 reported cases of angiocentric glioma. ${ }^{2-4,6,8,9}$ Of the reported cases, patient age at surgical treatment of these tumors ranged from 2 to 70 years old; however, with a median age of 16.0 \pm 14.3 years, it is evident that the disease predominantly affects children and young adults. ${ }^{6}$ The male/female ratio was approximately $1.5: 1$, and of the 59 patients in whom clinical features were described, $88 \%$ presented with intractable epilepsy. A majority of the cases shared common imaging findings including hyperintensity on T2-weighted and FLAIR MRI sequences as well as no contrast enhancement after gadolinium administration. ${ }^{4,6}$ The characteristic histological feature of angiocentric glioma is an angiocentric growth pattern of bipolar spindle cells. The tumor cells are immunoreactive for GFAP and vimentin, but negative for neuronal antigens and IDHIR $132 \mathrm{H}$ muta- 

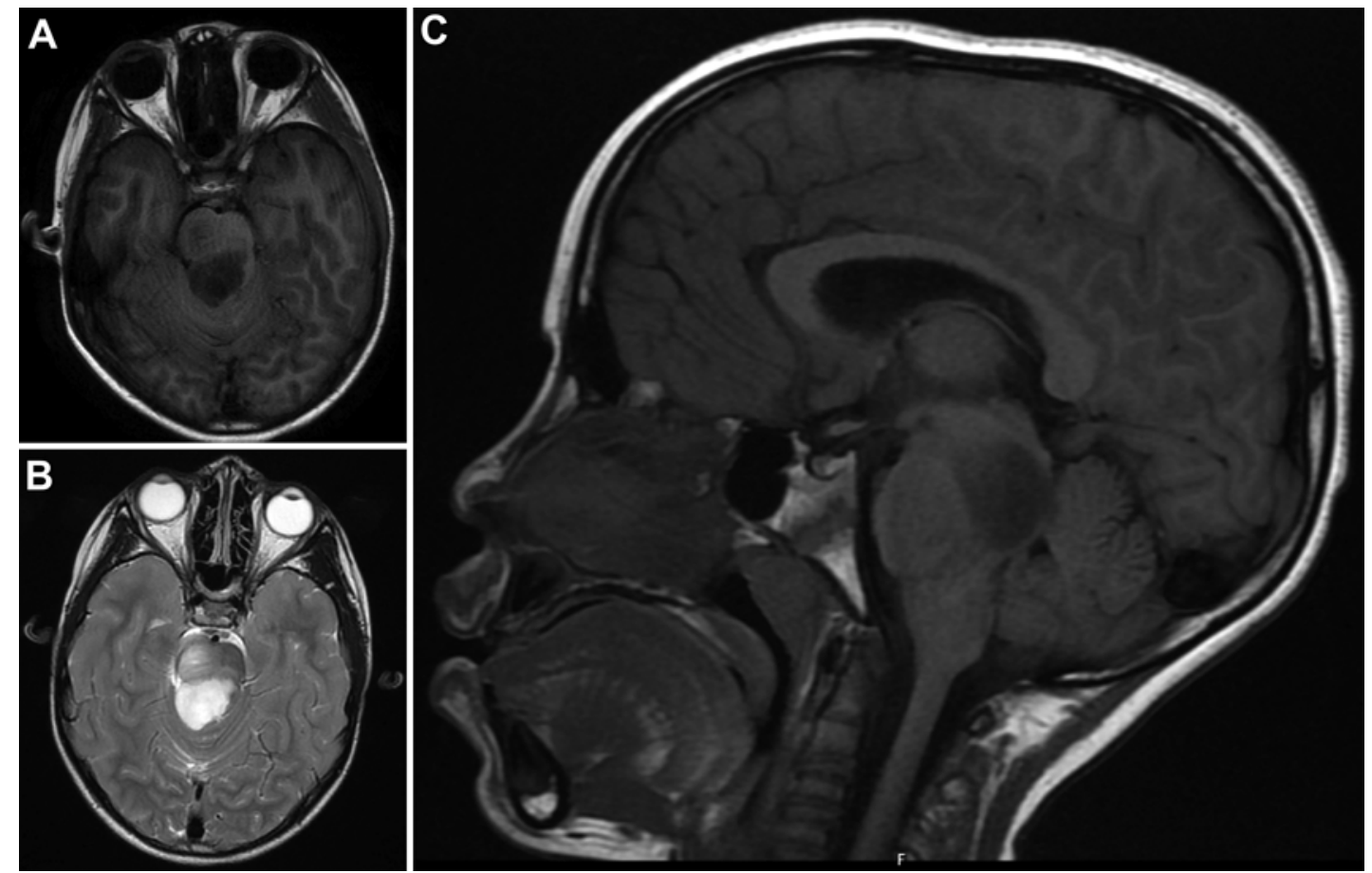

FIG. 4. Case 2. A: Axial T1-weighted MR image without contrast demonstrating an exophytic lesion of the pons with low T1 signal. B: Axial T2-weighted MR image demonstrating high T2 signal of the pontine lesion. C: Sagittal T1-weighted MR image.

tion. ${ }^{6,7}$ A majority of the tumors exhibit a dot-like perinuclear cytoplasmic staining for EMA, which is consistent with the ependymomatous features of angiocentric gliomas. ${ }^{6}$ In addition, angiocentric glioma classically exhibits a low proliferation index, with $89 \%$ of the reported cases having a Ki-67 index of $\leq 5 \%{ }^{6}$ Clinically, these tumors

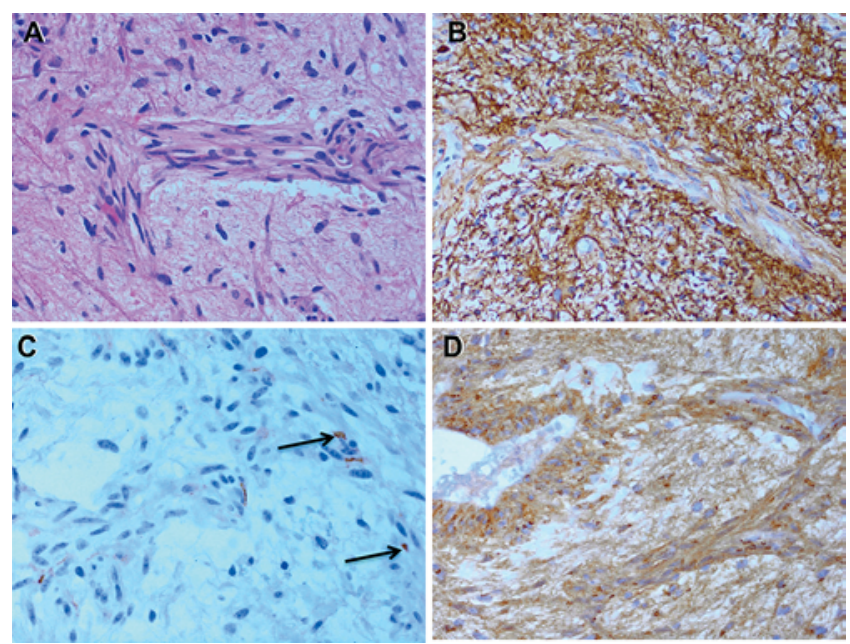

FIG. 5. Case 2. A: Photomicrograph showing a distinctive "angiocentric" pattern with no evidence of nuclear anaplasia. B: Photomicrograph showing the tumor cells displaying strong positivity for GFAP. C: Photomicrograph showing dot-like positivity for EMA in tumor cells (arrows). D: Photomicrograph obtained after staining for CD99 also showing perinuclear dot-like positivity in tumor cells. Original magnification $\times 400$. Figure is available in color online only. have continued to demonstrate indolent behavior as originally described in the 2007 WHO report. Even with subtotal resection, patients have done remarkably well, with many patients living seizure-free lives after surgery. ${ }^{6}$ There is only 1 case reported in which the patient experienced recurrence with an anaplastic (WHO Grade III) lesion and died shortly thereafter. ${ }^{9}$

Angiocentric gliomas almost exclusively involve tumors of the cerebral hemispheres. Prior to this case report, there was only 1 case reported of an angiocentric glioma involving the brainstem. In 2009, Covington et al. described the case of a 5-year-old girl who presented with gait instability and subtle cranial neuropathies and was found to have an exophytic midbrain tumor and obstructive hydrocephalus. ${ }^{3}$ The patient underwent ETV and craniotomy with an interhemispheric transtentorial approach for resection. Surgeons achieved a subtotal resection of the tumor without causing any new neurological deficits. Pathological analysis of the tumor specimen revealed monomorphous bipolar spindle cells with GFAP immunoreactivity and dot-like cytoplasmic staining for EMA. After 2 years of follow-up with serial imaging, the small amount of residual tumor showed no sign of progression.

In this report, we described the unique cases of 2 pediatric patients with angiocentric gliomas involving the brainstem. Both tumors exhibited the characteristic radiographic and histological features of angiocentric gliomas but were unique in their location and presentation. Our patients were treated differently, one with ETV and resection and the other with ETV and serial imaging to monitor for tumor progression. Both patients have remained stable over a period of several years, supporting the indolent 
course of this disease. In addition, the first case in this report included 6 years of follow-up for our patient whose case was very similar to that presented by Covington et al. ${ }^{3}$ This extended follow-up further supports an indolent course to this disease and augments the original knowledge gained from the 2 years of follow-up reported by Covington et al.

In summary, the cases described in this report further support the need, as first suggested by Covington et al., ${ }^{3}$ for inclusion of angiocentric glioma in the differential diagnosis of brain neoplasms occurring outside the cerebral hemispheres and presenting with symptoms other than refractory epilepsy. Brainstem tumors with features consistent with a diagnosis of angiocentric glioma may represent a distinct entity within the spectrum of low-grade gliomas affecting the brainstem and/or posterior fossa in the pediatric population. The location of these tumors presents a challenge to the neurosurgeon in terms of resectability; however, the cases described in this report as well as the case described by Covington et al. reveal positive clinical outcomes even with near-total or subtotal resection of the tumor. These outcomes support an indolent nature of brainstem angiocentric gliomas similar to angiocentric gliomas of the cerebral hemispheres. The first patient in this report has 6 years of follow-up; however, continued long-term follow-up of both patients is needed to ensure that the tumors continue to behave indolently.

\section{References}

1. Brat DJ, Scheithauer BW, Fuller GN, Tihan T: Newly codified glial neoplasms of the 2007 WHO Classification of Tumours of the Central Nervous System: angiocentric glioma, pilomyxoid astrocytoma and pituicytoma. Brain Pathol 17:319-324, 2007

2. Buccoliero AM, Castiglione F, Degl'innocenti DR, Moncini D, Spacca B, Giordano F, et al: Angiocentric glioma: clinical, morphological, immunohistochemical and molecular features in three pediatric cases. Clin Neuropathol 32:107-113, 2013

3. Covington DB, Rosenblum MK, Brathwaite CD, Sandberg DI: Angiocentric glioma-like tumor of the midbrain. Pediatr Neurosurg 45:429-433, 2009

4. Lellouch-Tubiana A, Boddaert N, Bourgeois M, Fohlen M, Jouvet A, Delalande O, et al: Angiocentric neuroepithelial tumor (ANET): a new epilepsy-related clinicopathological entity with distinctive MRI. Brain Pathol 15:281-286, 2005

5. Louis DN, Ohgaki H, Wiestler OD, Cavenee WK (eds): WHO Classification of Tumours of the Central Nervous System. Lyon, France: IARC, 2007

6. Ni HC, Chen SY, Chen L, Lu DH, Fu YJ, Piao YS: Angiocentric glioma: a report of nine new cases, including four with atypical histological features. Neuropathol Appl Neurobiol 41:333-346, 2015

7. Raghunathan A, Olar A, Vogel H, Parker JR, Coventry SC, Debski R, et al: Isocitrate dehydrogenase $1 \mathrm{R} 132 \mathrm{H}$ mutation is not detected in angiocentric glioma. Ann Diagn Pathol 16:255-259, 2012

8. Shakur SF, McGirt MJ, Johnson MW, Burger PC, Ahn E, Carson BS, et al: Angiocentric glioma: a case series. J Neurosurg Pediatr 3:197-202, 2009

9. Wang M, Tihan T, Rojiani AM, Bodhireddy SR, Prayson RA, Iacuone JJ, et al: Monomorphous angiocentric glioma: a distinctive epileptogenic neoplasm with features of infiltrating astrocytoma and ependymoma. J Neuropathol Exp Neurol 64:875-881, 2005

\section{Disclosures}

The authors report no conflict of interest concerning the materials or methods used in this study or the findings specified in this paper.

\section{Author Contributions}

Conception and design: Weaver, Pincus. Acquisition of data: Weaver, Bennett, Rivera-Zengotita. Analysis and interpretation of data: Weaver, Pincus. Drafting the article: Weaver, Crawford, Pincus. Critically revising the article: all authors. Reviewed submitted version of manuscript: all authors. Approved the final version of the manuscript on behalf of all authors: Weaver.

\section{Supplemental Information}

\section{Previous Presentations}

Portions of this work were presented in poster form at the 2014 AANS/CNS Section on Pediatric Neurological Surgery Annual Meeting, Amelia Island, Florida, December 2-5, 2014.

\section{Correspondence}

Kristin Weaver, Department of Neurosurgery, University of Florida, PO Box 100265, Gainesville, FL 32610. email: kristinjweaver@gmail.com. 